\title{
IMPACTO DEL ESTRÉS HÍDRICO Y LA PROCEDENCIA DE EUCALYPTUS GLOBULUS LABILL. SOBRE EL COMPORTAMIENTO DE HERBIVORÍA DE ACROMYRMEX LUNDI GUÉRIN
}

\author{
STRESS HYDRIC IMPACT AND THE PROVENANCE \\ OF EUCALYPTUS GLOBULUS LABILL. ON THE HERBIVORY \\ BEHAVIOR OF ACROMYRMEX LUNDI GUÉRIN
}

Patricia Caffarini ${ }^{1}$; Alicia Pelicano ${ }^{1}$; Paola Carrizo $^{1}$; Jorge Hugo Lemcoff ${ }^{1}$

\begin{abstract}
RESUMEN
Las diferentes especies de hormigas aceptan la mayor parte de las plantas que se les ofrecen; sin embargo, ha sido probado cierto comportamiento selectivo y en plantas bajo estrés hídrico se ha reportado mayor daño por su herviboría. Dos ensayos fueron llevados a cabo con la hormiga negra común, sobre siete procedencias de tres subespecies de Eucalyptus globulus. Tales procedencias fueron ofrecidas en nido artificial en laboratorio y bajo estrés hídrico, en nidos a campo. En laboratorio, la preferencia expresada como tiempo de acarreo fue significativamente diferente entre procedencias, aunque no entre subespecies. En campo, el área promedio removida fue significativamente mayor en plantas sometidas a estrés hídrico. Este pareció incrementar la atracción para las hormigas cortadoras, superando la preferencia relativa por las procedencias, manifestada en laboratorio. Por lo tanto, la utilización de procedencias menos preferidas es una alternativa de manejo sólo en aquellas áreas donde el estado hídrico puede ser controlado. La heterogeneidad del suelo causaría áreas relativamente más secas y tales situaciones localizadas de estrés hídrico podrían tornar a ciertos individuos más atractivos y, por lo tanto, más dañados.
\end{abstract}

Palabras clave: Estrés, hídrico, Acromyrmex lundi, Eucalyptus globulus, herbivoría.

\section{ABSTRACT}

The different ant species, forage most of the plants offered to them. However, a kind of selective behavior has been proven among them. In addition, in plants underwater stress, a larger herbivory damage has been reported. Two experiments were carried out with the black common cutting ant, on seven provenences of three subspecies of Eucalyptus globulus. Provenances were first tested in trials in an artificial nest in laboratory conditions. Later, the same provenances were taken outdoors, to natural nests, using controlled and water stressed seedlings. In lab trials, carrying time was significant different among provenances, although not among subspecies. In the open field, average leaf area removed was significantly larger for the stressed plants. It seemed water stress made the eucalypt seedlings more attractive to cutting ants this effect over the provenance. Thus, utilization of less preferred provenances will be a technical alternative in places where the plant water status can be controlled. Under outdoor conditions, soil heterogeneity could reveal areas drier than others; this could change herbivory preference from ants, and cause them higher damage.

Key words: Water stress - Acromyrmex lundi, Eucalyptus globulus, herbivory damage.

\section{INTRODUCCIÓN}

Las plantas sometidas a estrés por sequía presentan una pérdida del color verde, tienen mayor temperatura foliar y reflectancia de luz infrarroja, características que las harían más aceptables o atractivas a los insectos (Mattson y Haack, 1987; Moore, 1995). Esto sería posible dado que los artrópodos poseen quimiorreceptores sensibles al estado hídrico de la planta y al nivel de aminoácidos, azúcares, sales y aleloquímicos libres (Städler, 1984; Mattson y Haack, 1987).

1 Facultad Agronomía, Universidad de Buenos Aires, Av. San Martín 4453 (1417). Buenos Aires, Argentina. e-mail: pelicano@agro.uba.ar

Fecha de Recepción: 29 Diciembre de 2005

Fecha de Aceptación: 17 Febrero de 2006 
En plantas de Eucalyptus camaldulensis sometidas a estrés hídrico, Stone y Bacon (1994) observaron mayor daño por herbivoría de insectos, aun cuando no detectaron alteraciones en los componentes químicos de las hojas. Por su parte, Della Lucia et al. (1994) y De Queiroz Santana y dos Anjos (1989) demostraron la existencia de preferencia diferencial en el ataque a eucalipto bajo estrés hídrico por hormigas cortadoras de los géneros Acromyrmex y Atta.

La herbivoría por hormigas cortadoras constituye un problema en el establecimiento del monte de eucalipto, ya que compromete el crecimiento y puede llegar a afectar la supervivencia de las plantas (Cozzo, 1995; Carpinetti et al., 1995; Della Lucia, 1993). Las distintas especies de hormigas forrajean la mayor parte de los vegetales ofrecidos (Queiroz Santana y Dos Anjos, 1989); sin embargo, se ha demostrado cierta preferencia (Mariconi, 1963; Souza, 1965; Wiemer y Ales, 1981; Hubbell et al., 1983).

La capacidad selectiva de las hormigas ha sido observada por Queiroz Santana y Dos Anjos (1989) y Caffarini et al., (2000), entre otros. Si el estrés hídrico influye en los fenómenos de herbivoría, alterando el comportamiento de selección, podría alterarse el estatus de esta plaga en campo.

En este contexto, el objetivo de este trabajo fue evaluar el impacto del estrés hídrico y la procedencia de Eucalyptus globulus sobre el comportamiento de herbivoría de Acromyrmex lundi.

\section{MATERIALES Y MÉTODOS}

Se realizaron dos experiencias con A. lundi en campo y laboratorio, con plantas de Eucalyptus de 6 meses de edad, de procedencias seleccionadas por resultados previos (De Haro et al., 2000). Procedencias:

- Eucalyptus globulus globulus: Jeerlanang, Seymour y Otaways National Park.

- E. globulus maidenii: Bolaro Mt. y Murrabrine.

- E. globulus bicostata: Nullo Mountain y Wee Jasper.

Las semillas se obtuvieron por importación desde las localidades australianas de referencia. Los plantines se realizaron en el campo experimental de la FAUBA.

\section{ENSAYO DE LABORATORIO. PREFERENCIA DE PROCEDENCIAS}

Se utilizó un hormiguero artificial (Della Lucia et al., 1993), formado por un conjunto de frascos de 3 litros de capacidad, conectados mediante mangueras plásticas entre sí y a una playa de forrajeo de $1.800 \mathrm{~cm}^{2}$ con paredes de vidrio. En los frascos se alojaba el hongo simbionte, las crías y la cámara de desechos -donde depositan los cadáveres, restos vegetales rechazados y trozos secos del hongo-.

La alimentación habitual de cría consistió en hojas de ligustrina (Ligustrum sinense Lour.), ligustro (Ligustrum lucidum Ait.), mora negra (Morus nigra L.) y albura de naranja (Citrus sinensis L.). La temperatura se mantuvo a $24 \pm 2{ }^{\circ} \mathrm{C}$, la humedad relativa a 70-80\% y el fotoperíodo 12:12 luz-oscuridad (Della Lucia et al., 1993).

Los ensayos se llevaron a cabo mediante pruebas sin libre elección con intervalos de 48 horas, durante los cuales se les proporcionó la dieta de cría. El diseño consistió en un DCA con 7 réplicas. Para cada procedencia se utilizaron 15 discos de $0,5 \mathrm{~cm}$ de diámetro, extraídos de hojas de la parte media. Este material vegetal fue dispuesto sobre papel de filtro húmedo, para evitar alteraciones en el contenido de agua.

Se midió el tiempo de acarreo para retirar todos los discos de cada procedencia (Vendramin et al., 1995). Los valores obtenidos se analizaron mediante ANOVA y Tukey (alfa $=0,05$ ) de los datos transformados mediante $\log _{10}$ (Sokal y Rohlf, 1969).

\section{ENSAYO DE CAMPO. PREFERENCIA DE PROCEDENCIAS Y EFECTOS DE ESTRÉS HIIDRICO}

Generación del estrés hídrico. Las plantas se mantuvieron en macetas de plástico negro $(6 \mathrm{~cm}$ de diámetro y $12 \mathrm{~cm}$ de alto), en una mezcla de tierra y arena $(3 \mathrm{~V} / 1 \mathrm{~V})$ como sustrato. El estrés hídrico fue generado por reducciones semanales progresivas (50 / 40 / 25 y 10\%) de los volúmenes de riego diario con respecto del requerido para capacidad de campo, determinado diariamente por pesaje de las macetas (Barrs \& Weatherley, 1962).

El contenido relativo de agua (CRA) para las plantas fue de $71 \%$ (A) y $64 \%$ (B) para los tratamientos de estrés y $92 \%$ (A) y $84 \%$ (B) para los controles. La restricción hídrica se mantuvo regando el $10 \%$ del volumen del control. 
Con las plantas estresadas y control así obtenidas, en hormiguero a cielo abierto se realizaron dos ensayos sucesivos. Los tratamientos fueron distribuidos en bloques al azar, donde el bloque representó cada uno de los caminos principales, hacia las bocas activas del hormiguero.

Las plantas estuvieron expuestas durante 15 días; al final de este período se evaluó la relación área foliar total removida / ofrecida, que fue estimada mediante la siguiente escala: $0,1 / 4,1 / 2,3 / 4$ y 1 , donde esta última implica que todas las hojas fueron removidas. Se realizó un ensayo en febrero, con 6 bloques $\left(23,5 \pm 2,2^{\circ} \mathrm{C}\right.$ y H.R. $\left.61 \pm 8 \%\right)$ y otro en marzo, con 3 bloques $\left(19,7 \pm 2,4^{\circ} \mathrm{C}\right.$ y H.R. $62 \pm 6 \%)$.

Los resultados se analizaron mediante la prueba de ANOVA de dos vías (Riego x Procedencia) y Tukey $(\mathrm{p}=0,05)$ de los datos transformados mediante arcsenÖx (Sokal y Rohlf, 1969).

\section{RESULTADOS Y DISCUSIÓN}

\section{ENSAYO EN LABORATORIO}

El análisis de varianza arrojó diferencias significativas $(\mathrm{p}<0,0001)$ para las procedencias, con respecto al tiempo requerido para remover todos los discos de eucalipto (Figura 1), sin presentar un patrón o tendencia asociada a las subespecies. La prueba de Tukey separó grupos sin relación con las subespecies.

\section{ENSAYO DE CAMPO}

En ambos ensayos, el área foliar removida fue significativamente mayor para las plantas sometidas a estrés hídrico ( $\mathrm{p}=0,0015$ en A y $\mathrm{p}=0,0002$ en B). No hubo un efecto de interacción ni debido a las subespecies (Figura 2a y 2b).

Asimismo, en el ensayo de febrero, el área removida en las plantas sin estrés o "control” (22,86 $\pm 2,82 \%$ ) fue menor que en el ensayo en marzo $(39,08 \pm 3,48 \%)$. Según Tukey, se observan 4 grupos con diferencias significativas entre las subespecies y/o procedencias en estudio. Con respecto a las procedencias, sólo se presentaron diferencias significativas en la subespecie globulus.

En las pruebas de laboratorio con procedencias de E. globulus, las hormigas cortadoras respondieron de modo diferencial. Estos resultados coinciden con aquellos de Queiroz Santana y Dos Anjos (1989), Santana y Couto (1990) y Vendramin et al. (1995).

Tales resultados podrían hacer posible caracterizar estos materiales como portadores de dife-

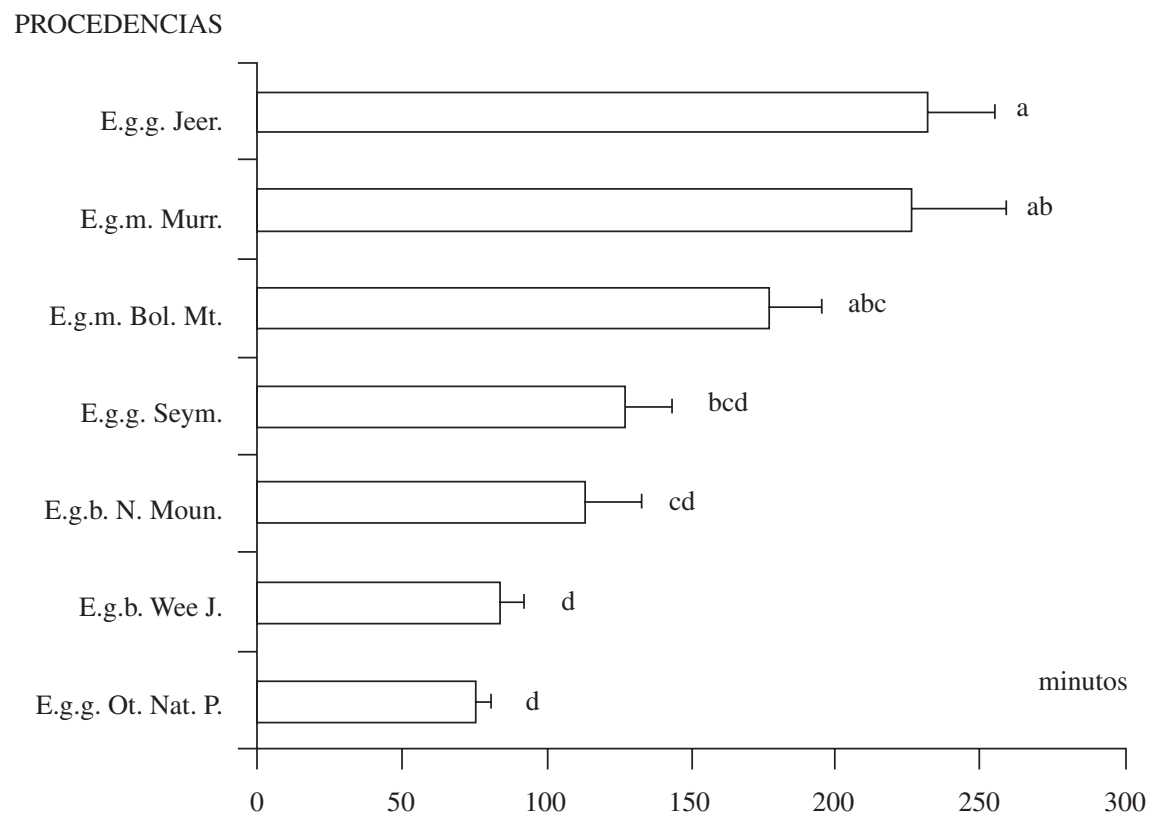

Figura 1. Tiempo de remoción promedio para las procedencias de Eucalyptus en laboratorio. Las barras indican el error estándar de la media. Las letras corresponden a la prueba de Tukey: letras diferentes implican diferencias significativas. 

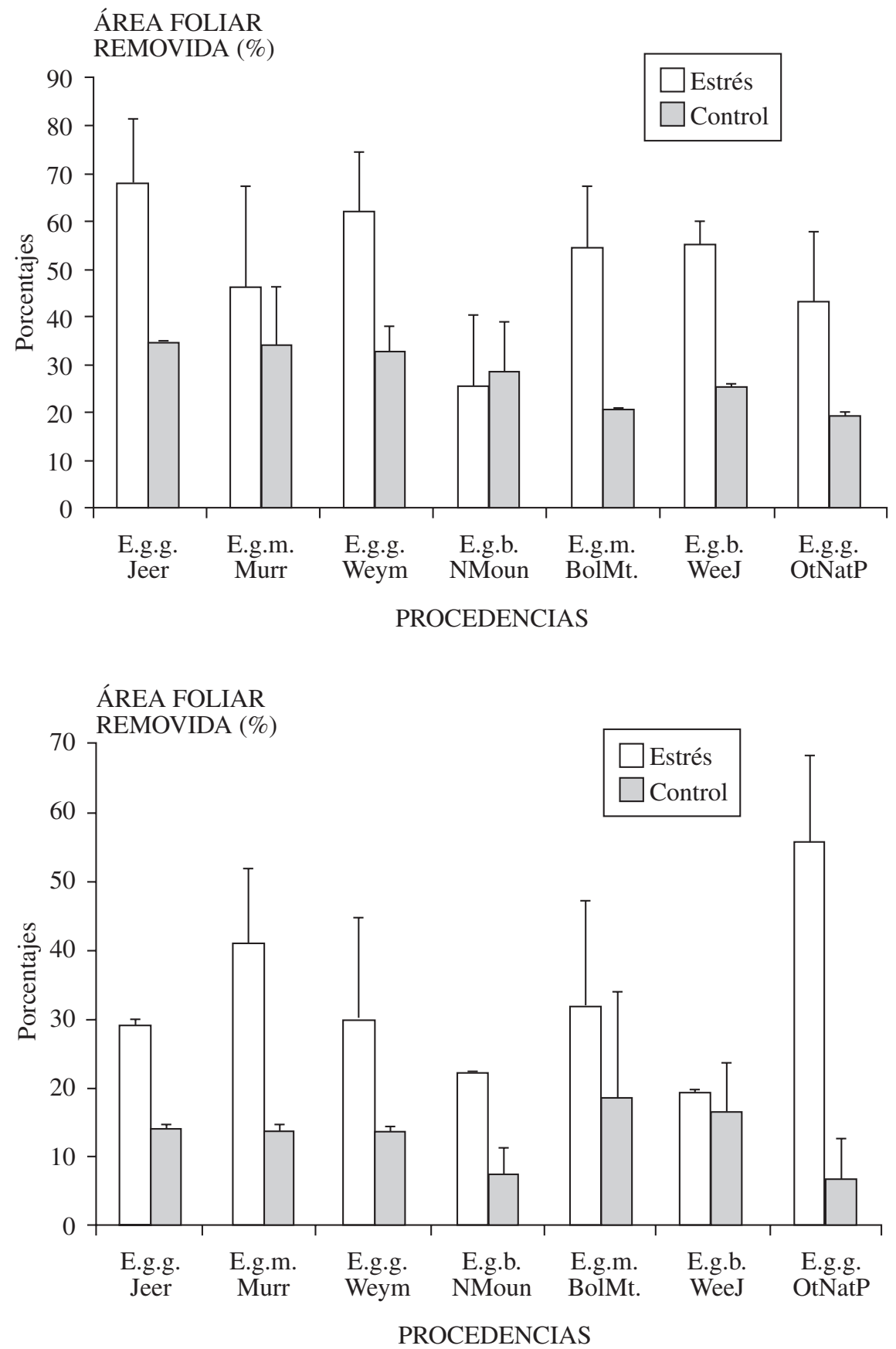

Figuras 2.a. y b. Primer y segundo ensayo en campo: Área foliar removida por procedencia. Las barras representan el error estándar de la media. 
rentes grados de tolerancia a herbivoría, de acuerdo con la visión clásica de la resistencia, según la entiende Painter (1951). De acuerdo con la Figura, estas procedencias podrían ser consideradas para la escala de tolerancia a herbivoría como: resistente (Jeerelang y Murrabrine); moderadamente resistente (Bolaro Mountain); moderadamente susceptible (Seymour y Nullo Mountain) y susceptible (Wee Jasper y Otaways National Park).

Bajo estrés hídrico, la proximidad genética entre las procedencias no permite hallar diferencias en los componentes químicos estables, según

\section{LITERATURA CITADA}

BARRS, H.; WEATHERLEY, P. 1962. A reexamination of the relative turgidity technique for estimating water deficits in leaves. Aust. J. Biol. Sci. 15: 413-428.

CAFFARINI, P.; PELICANO, A.; CARRIZO, P.; LEMCOFF, J. 2000. Water stress effect on worker cutting-ants Acromyrmex hispidus on three origins of Eucalyptus globulus globulus. En: Proc. XXI Int. Cong. Entomol. Brasil.

CARPINETTI, L.; DALLA TEA, F.; GLADE, J.; MARCÓ M. (EDS.) 1995. Manual para productores de eucaliptos de la Mesopotamia Argentina. SAGyP-INTA. 162 p.

COZZO, D. 1995. Silvicultura de plantaciones maderables. T 2. Orientación Gráf. Ed.

DE HARO, A.; BARAÑAO, J.; MARTÍNEZ, C.; RIQUELME, M. 2000. Actas XXII Congreso Nacional de Entomología. Valdivia, Chile.

DELLA LUCIA, T. (ED.) 1993. As formigas cortadeiras. Vicosa, Mina Gerais, Brasil.

DELLA LUCIA, T.; DE OLIVEIRA, M.; DA SILVA ARAUJO, M.; FERREIRA VILELA, E. 1994. Screening for non-preference of the leaf-cutting ant Acromyrmex subterraneus for Eucalyptus. Rev. Arvore 19: 92-99.

HUBBELL, S. P.; WIEMER D. F.; ADEJARE A. 1983. An antifungal terpenoid defends a neotropical tree (Hymenae) against attack by fungus growing ants (Atta). Oecologia, (Berlim) 60: 321-327.

MATTSON, W.; HAACK, R. 1987. The role of drought in the outbreak of the plant-eating insects. Bioscience 37: 110-118.

MARICONI, F. A. M. 1963. Insecticidas e seu emprego no combate às pragas. São Paulo: Agronômica Ceres. 607 p.
Stone y Bacon (1994) para E. camaldulensis. Algo similar puede observarse en el presente trabajo, donde el efecto del estrés hídrico prevaleció sobre las diferencias debidas a las procedencias, de modo similar a lo observado por Mattson and Haack (1987) and Moore (1995).

Bajo condiciones de campo, la heterogeneidad del suelo podría generar áreas relativamente más secas. Estas podrían, asimismo, influir en la preferencia relativa por ciertos individuos sobre otros y en consecuencia producir mayores daños en sitios particulares. Sin embargo, sería menester validarla en situación de cultivo comercial.

MOORE, P. D. 1995. How plants deal with what's eating them. Science Watch, 6: 7-8.

PAINTER, R. H. 1951. Insect resistance in crop plants. New York, Mc Millan.

QUEIROZ SANTANA M.; DOS ANJOS, N. 1989. Resistencia de Eucalyptus spp. (Mirtaceae) a Atta sexdens rubropilosa e Atta laevigata. Rev. Arvore 13, 2: 174-181.

SANTANA, D. L.; COUTO, L. 1990. Resistencia intra-específica de eucaliptos a formigas cortadeiras. Bol. Pes. Flor. Curitiba, 20: 13-21.

SOKAL, R. R.; ROHLF, F. J. 1969. Biometry. W. H. Freedmand \& Co., San Francisco, USA. 776 p.

SOUZA L. F. 1965. Plantas preferidas pela saúvas-limão. Divulgação Agronômica, Rio de Janeiro, 14: 23-29.

STÄDLER, E. 1984. Contact chemoreception. Pág. 3-35. En W. J. Bell y R. Carde, editores. Chemical Ecology of Insects. Sinauer Assoc. Suntherland, MA. 612 p.

STONE, C.; BACON, P. 1994. Relationships among moisture stress, insect herbivory, foliar cineole content and the growth of river red gum Eucalyptus camaldulensis. J. Appl. Ecol. 31: 604-612.

VENDRAMIN, J. V.; SILVEIRA NETO, S.; CERIGNONI, J. A. 1995. Nao-preferencia de Atta sexdens rubropilosa forel por especies de Eucalyptus. Eccosistema 20: 87-92.

WIEMER, D. F.; ALES, D. C. 1981. Lasidiol angelate: Ant repellent sesquiterpenoid from Lasiantheae fruticosa. Journal of Organic Chemistry, Baltimore 46: 5449-5450. 